\title{
Influence of Blade Thickness Distribution on the Structural Dynamic Characteristics Under Part-Load Condition
}

\author{
Hao CHANG, Wei LI*, Weidong SHI**, Lei WANG, Jianrui LIU \\ *Research Center of Fluid Machinery Engineering and Technology, Jiangsu University, Zhenjiang 212013, China, \\ E-mail: lwjiangda@ujs.edu.cn \\ **Nantong University, Nantong 226019, China, E-mail: Wdshi2012@126.com \\ crossref http://dx.doi.org/10.5755/j01.mech.25.1.22882
}

\section{Introduction}

Blades as the core part of the pump, which can not only influence the hydraulic performance, but also affect the strength of the impeller. In recent year, plenty of the efforts have been taken in the blade performance optimization. Shigemitsu [1,2] investigated the influence of the blade outlet angle on the internal flow field, the test result shows that the head is increased by rising the blade outlet angle, while the maximum efficiency gradually decreases $\mathrm{Li}$ [3] assessed the cavitation performance of engine cooling water pump, and analyzed the effect of the blade outlet widths via numerical simulation. Wang [4] selected blade outlet width, impeller diameter, number of blades and exit blade angle as target factors, by employing the orthogonal experiment to optimize the external characteristic. Chakraborty [5] simulated the centrifugal pump with different blade number to improve the internal flow field characteristics. Lei [6] presented the relationship between the blade warp angle and the hydraulic performance based on the direct and inverse iteration design method. Derakhshan [7] combined the genetic algorithm and artificial neural network, and obtained the optimal parameters of camber line in blade. Wang [8] designed four different inlet angle impellers, and founded the best efficiency point can be improved by increasing the blade inlet angle. Jeon [9] introduced the three-dimensional NavierStokes analysis and the response surface method, analyzed the effect of the upper blade height and hub height on the efficiency. Tan [10] studied the influence of blade rotational angles on the energy performance and pressure fluctuation characteristics of the mixed-flow pump.

However, most previous analyses concentrate on the modification of the blade geometry parameters at the design flow rate, only a few researches improve the performance by changing the blade thickness distribution. In this paper, blades were designed based on the empirical correlation equation, which according to plenty of outstanding hydraulic models. And four blades with different thickness distribution were proposed based on the previous research [11]. And then, by comparing the modal properties, strength characteristics and hydraulic performance under 0.2 times flow rate condition, the optimal blade thickness distribution were obtained. Finally, the test was conducted to benchmark the accuracy of the simulation results. This research could provide reference for designing the thickness distribution of the blade.

\section{Numerical investigation}

\subsection{Computational domain}

The design specifications of the self-priming pump are shown as follow: $Q=500 \mathrm{~m}^{3} / \mathrm{h}$, head $H=45 \mathrm{~m}$ and rotation speed $n=2200 \mathrm{r} / \mathrm{min}$. The computational domains of the self-priming pump include five parts: inlet, self-priming system, impeller, volute and outlet. Four times of pipe diameter were extended in the inlet and outlet to get the stable incoming flow and fully development outflow, as shown in Fig. 1.

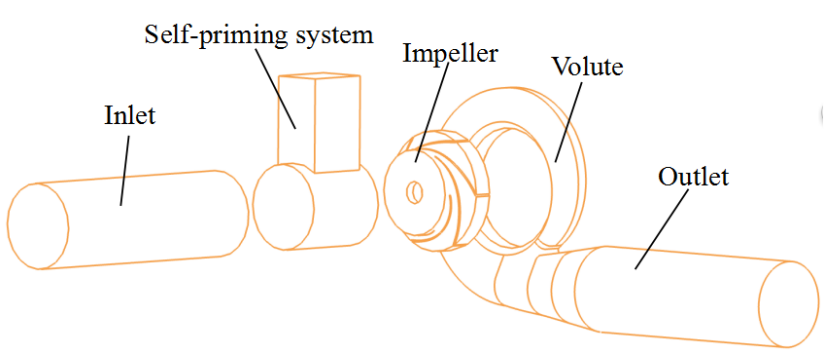

Fig. 1 Computational domains

\subsection{Grid generation}

In this paper, the computational domain was discretized into structured grids by ICEM CFD. Fig. 2 presents the analysis of grid independency, when the number of the elements exceeded 2671710, the fluctuation of the head is less than $1 \%$. The suitable elements number of inlet, outlet, volute, impeller and suction casing of self-priming system are 504000, 696000, 720480, 478998 and 272232, respectively (Fig. 3).

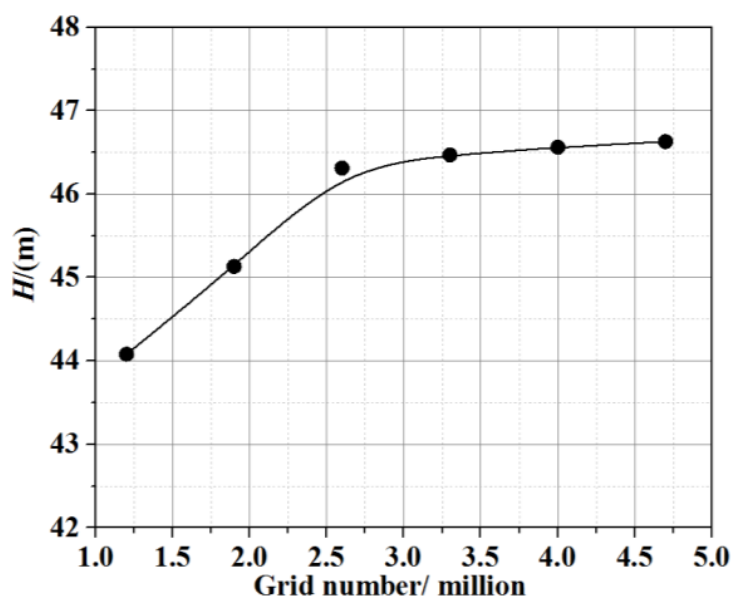

Fig. 2 Grid independency analysis 
The blades were designed according to the empirical correlation equation, which is obtained by plenty of the outstanding hydraulic models. And the four different thickness blades were proposed based on the previous research [11]. Table 1 lists the thickness distribution from the leading edge to the trailing edge of the blade.

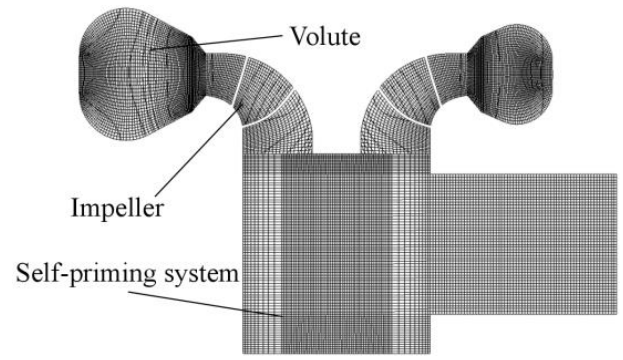

Fig. 3 Structured grid of computational domain

Table 1

Thickness distribution of each scheme

\begin{tabular}{|c|c|c|c|}
\hline Scheme & $\begin{array}{c}\text { Thickness of } \\
\text { leading edge } \\
(\mathrm{mm})\end{array}$ & $\begin{array}{c}\text { Thickness of } \\
\text { middle part } \\
(\mathrm{mm})\end{array}$ & $\begin{array}{c}\text { Thickness } \\
\text { of trailing } \\
\text { edge }(\mathrm{mm})\end{array}$ \\
\hline 1 & 3 & 3 & 3 \\
\hline 2 & 6 & 6 & 6 \\
\hline 3 & 3 & 6 & 6 \\
\hline 4 & 6 & 6 & 3 \\
\hline
\end{tabular}

\subsection{Simulation method}

The steady simulation is carried out by applying SST $k$ - $\omega$ turbulence model in ANSYS CFX, and the water at $25^{\circ} \mathrm{C}$ as the flow media. The impeller is set as rotational computational domain, while the other domain set as stationary. Meanwhile, the interfaces between the stationary domain and rotational domain are set as the frozen rotor interfaces, and the interfaces between stationary domains are set as general grid. Furthermore, the roughness of wall is $50 \mu \mathrm{m}$, the boundary condition is selected as the total pressure inlet and mass flow outlet. Additionally, convergence of the residuals is 0.00001 , the high resolution is employed as advection scheme.

The structural dynamics is employed to analyze the strength of the impeller. The internal flow field of the steady simulation is loaded on the blade surface, inner surface of the shroud and hub. Further, the gravity and rotating centrifugal force are added upon the impeller. The structure of impeller is discretized into unstructured grids by ANSYS Workbench, as shown in Fig. 4. The number of the elements is 154702 , and the node reaches 256262 .

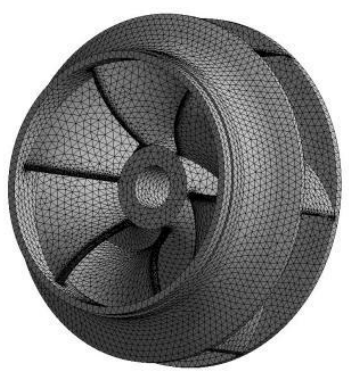

Fig. 4 Unstructured grids of the impeller

\subsection{Structural dynamic equation}

\subsubsection{The Structural dynamic equation in air}

The dynamic equation with $\mathrm{n}$ degree freedom system as follow:

$$
M \ddot{x}+C \dot{x}+K x=F,
$$

where: $M, C, K$ are the matrix of mass, damping and stiffness, respectively; $x, \dot{x}, \ddot{x}$ are the matrix of the displacement, velocity and acceleration; $F$ is the matrix of external excitation force.

When the modal properties are investigated, the structure under the free vibration condition, namely, $C=0$, the basic dynamic equation of the modal properties as follow:

$$
M \ddot{x}+K x=0 .
$$

The form of the solution equation is obtained:

$$
x=X \sin (p t+h) .
$$

Characteristic determinant of system is calculated as:

$$
\left|K-p^{2} M\right|=0
$$
obtained.

Finally, the natural frequency of each mode can be

\subsubsection{The Structural dynamic equation in water}

The Eq. (2) can only solve the modal properties in the air, but the impeller actually rotating in the water, the interaction is generated between fluid and structure. What is more, when the vibration is created on the impeller, under the effect of viscous force and inertia force, the fluid attached on the impeller vibrates together with the impeller. Therefore, the total mass involves the vibration is increased, which results in the vibration distribution of the impeller has changed. Meanwhile, with the vibration of the water, the energy loss is generated in the internal flow. Hence, considering the effect of the water, the pressure is set as the unknown variable to solve the modal properties of the impeller submerged in water.

Water compressibility wave equation is calculated as:

$$
\frac{1}{c} \frac{\partial^{2} p}{\partial^{2} t^{2}}-\Delta^{2} p=0 .
$$

Discretizing the Eq. (5):

$$
M_{f} \ddot{p}+K_{f} p=F_{f} \text {. }
$$

Eq. (6) is substituted into Eq. (1):

$$
\left[\begin{array}{cc}
M & 0 \\
\rho_{f} R & M_{f}
\end{array}\right]\left[\begin{array}{l}
\ddot{x} \\
\ddot{p}
\end{array}\right]+\left[\begin{array}{cc}
K+K_{r} & R \\
0 & K_{f}
\end{array}\right]\left[\begin{array}{l}
x \\
p
\end{array}\right]=\left[\begin{array}{l}
F_{s} \\
F_{f}
\end{array}\right],
$$


where: $c$ is the velocity of sound in water, $M_{f}, \rho_{\mathrm{f}}$ and $K_{f}$ are the matrix of mass, density and stiffness of additional water; $F_{s}, F_{f}$ are the excitation force matrix of structure and additional water; $p$ and $\ddot{p}$ are the matrix of the particle displacement and acceleration; $R$ is the conditional matrix of fluidstructure interaction; $K_{r}$ is the stiffness matrix of centrifugal force stress.

\section{Results and discussions}

\subsection{Analysis of the energy performance}

Fig. 5 shows the energy performance curve of each scheme, it can be found that the energy performance of the thin blade (scheme 1) is larger than uniform thickening blade (scheme 2) under the part-load condition $\left(100 \mathrm{~m}^{3} / \mathrm{h}\right.$ ), while the energy performance of the increment thickness distribution blade (scheme 3 ) reaches the maximum. That is, the energy performance of the part-load condition cannot be improved by applying the uniform thickening on blade, but the local thickening blade can be achieved. Meanwhile, the energy loss of the increment blade is lower than the decrement blade.

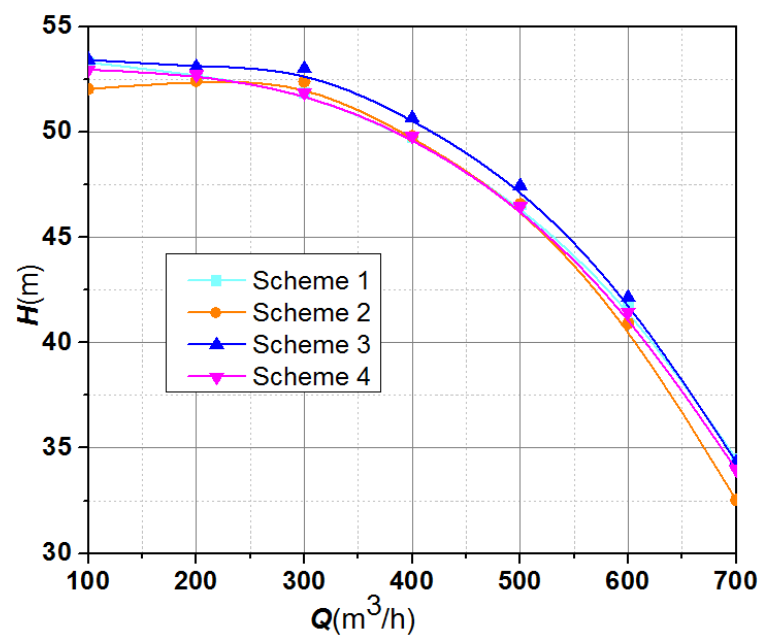

a) The head curve under different flow rates

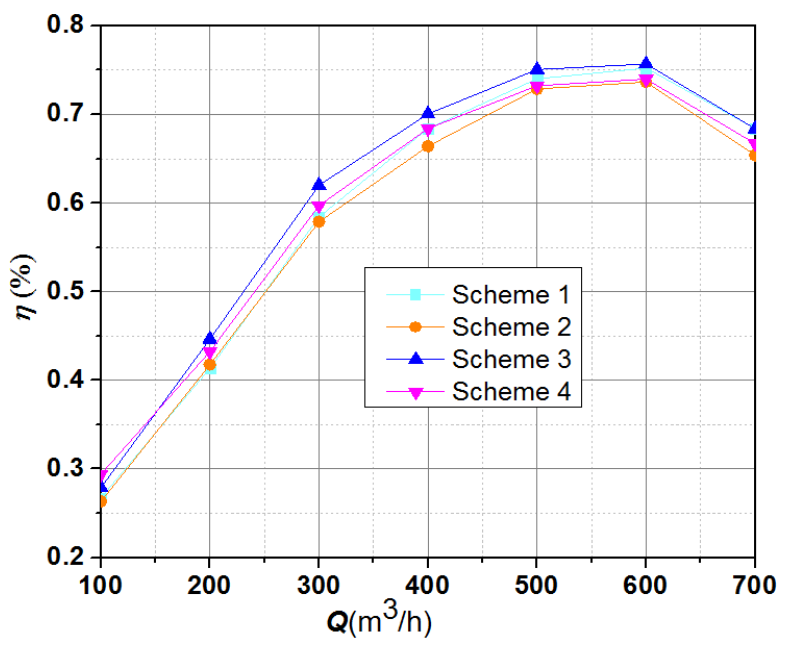

b) The efficiency curve under different flow rates

Fig. 5 Energy performance curves of each scheme

\subsection{Pressure distribution}

Fig. 6 presents the pressure distribution of pump under $0.2 Q$, owing to the work of blade, the pressure is increasing along the flow direction, and reaches the maximum at the outlet of volute. However, due to the difference of the blade, the area of the high pressure at the volute is different. It can observe that the area of high pressure by employing the uniform thickening blade is small, while the area is increasing by applying the local thickening blade. Furthermore, the high pressure area of the scheme 3 is larger than the scheme 4 , that is, the internal flow field can be improved by using increment thickness distribution blade, which is benefit to transform the kinetic energy into the potential energy.

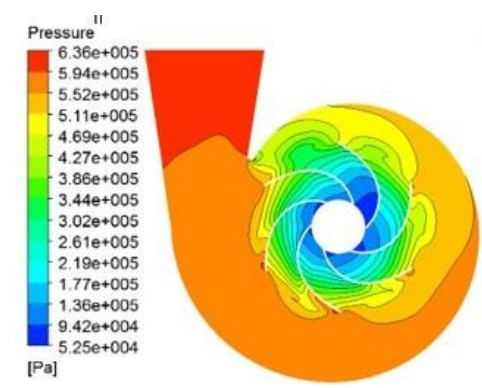

Scheme 1

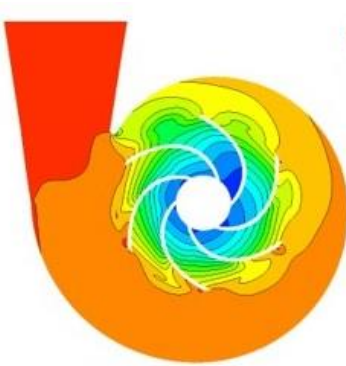

Scheme 3

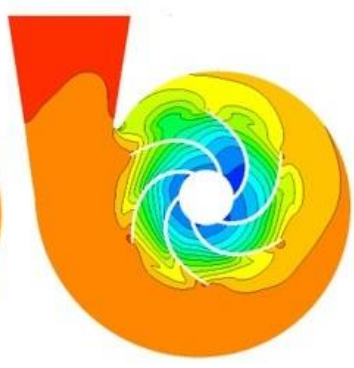

Scheme 2

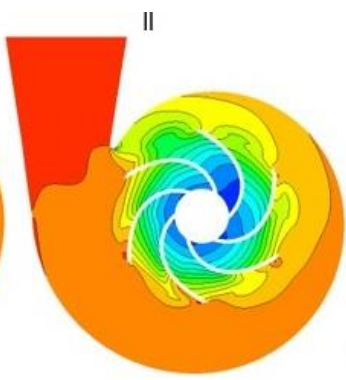

Scheme 4
Fig. 6 Pressure distribution of pump under $0.2 Q$

\subsection{Structural dynamic characteristics}

\subsubsection{Modal properties}

In order to avoid the vibration induced by resonance and ensure the self-priming pump can operate in a stability condition, the natural frequency of the blades submerged in water are analyzed, as shown in Fig. 7. It found that the natural frequency increase smoothly within first three modes, but rise sharply under the fourth mode. Meanwhile, the growth of natural frequency under last three modes is slight. By comparing the natural frequency of schemes with different thickness distribution, it can be seen that the natural frequency of each scheme have the similar variation trend. The natural frequency of the scheme 2 with the uniform thickening blade is remarkable lager than other schemes, while the natural frequency of the scheme 1 with thinning blade reaches the minimum. At the same time, the natural frequency of the scheme 4 is larger than the scheme 3 within first four modes, but reaches the same value at the fifth and sixth mode. Due to the structural stiffness is improved by applying the thickening blade, the natural frequency of the thicker blade is obviously enhanced, and the 
natural frequency of the blade with decrement thickness distribution is larger than the blade with increment thickness distribution, namely, thickening the blade leading edge is benefit to improve the natural frequency of the impeller. Considering the rotation speed of the impeller is $2200 \mathrm{r} / \mathrm{min}$, the number of blades is 6 , therefore, the shaft natural frequency and the blade passing natural frequency can be obtained: shaft natural frequency is $36.7 \mathrm{~Hz}$ and the blade passing natural frequency is $220 \mathrm{~Hz}$, which is much lower than the natural frequency of the first mode, so it can effectively avoid the occurrence of resonance.

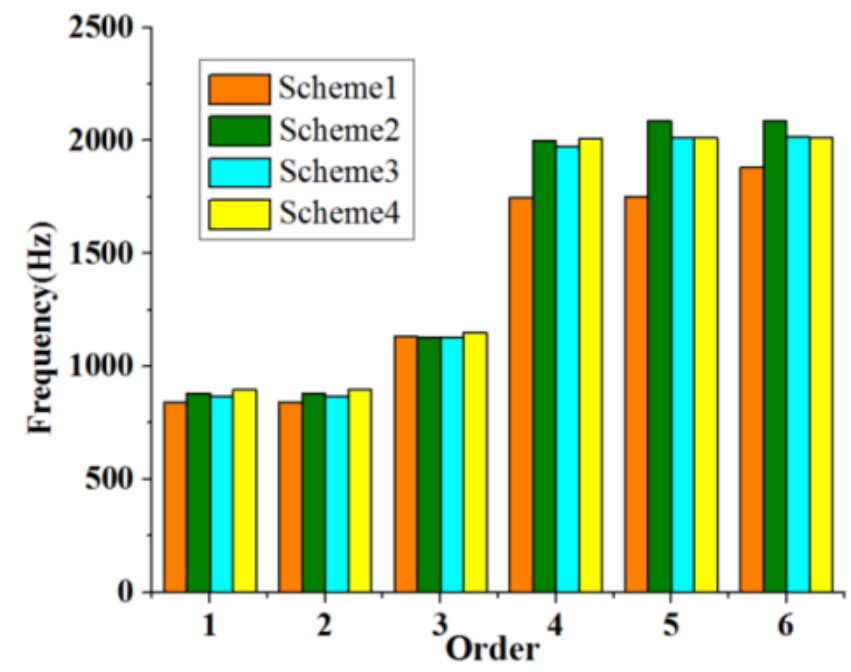

Fig. 7 Natural frequency of the blades under $0.2 Q$
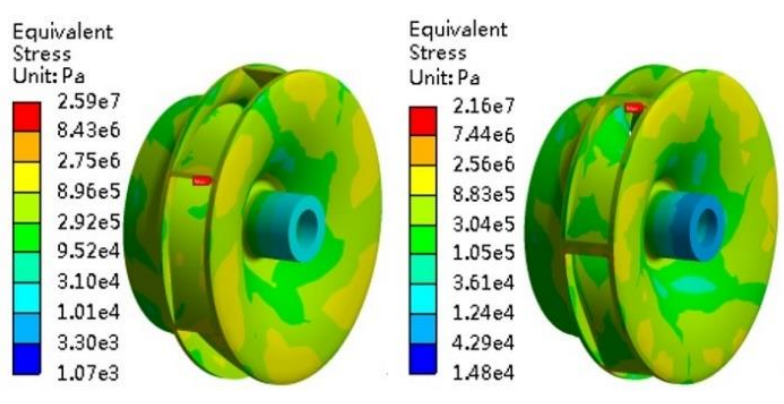

Fig. 8 Equivalent stress distribution under $0.2 Q$

Total
Deformation

Unit: $\mathrm{mm}$

$\square \begin{aligned} & 2.61 \mathrm{e}-2 \\ & 2.32 \mathrm{e}-2\end{aligned}$

$2.32 \mathrm{e}-2$
$2.03 \mathrm{e}-2$

$2.03 \mathrm{e}-2$
$1.74 \mathrm{e}-2$

$1.45 \mathrm{e}-2$

$1.16 \mathrm{e}-2$

$8.70 \mathrm{e}-3$

$5.80 \mathrm{e}-3$

$2.90 \mathrm{e}-3$

$0.00 \mathrm{e} 0$

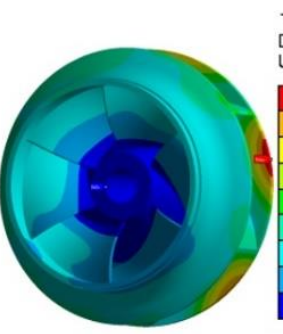

Total

Deformatio

Unit: $\mathrm{mm}$
$1.19 \mathrm{e}-2$

$1.19 \mathrm{e}-2$
$1.06 \mathrm{e}-2$

$9.26 \mathrm{e}-3$
$7.94 \mathrm{e}-3$

$7.94 \mathrm{e}-3$

$6.62 \mathrm{e}-3$

$5.29 \mathrm{e}-3$
$3.97 \mathrm{e}-3$

$3.97 \mathrm{e}-3$

$2.65 \mathrm{e}-3$

$1.32 \mathrm{e}-3$
$0.00 \mathrm{e} 0$

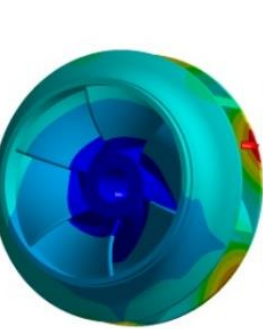

Deformatio

$2.33 \mathrm{e}-2$

$2.33 \mathrm{e}-2$
$2.07 \mathrm{e}-2$

$-\begin{aligned} & 1.81 \mathrm{e}-2 \\ & 1.55 \mathrm{e}-2\end{aligned}$

$1.55 \mathrm{e}-2$
$1.29 \mathrm{e}-2$

$1.29 \mathrm{e}-2$
$1.04 \mathrm{e}-2$

$7.77 \mathrm{e}-3$

$5.18 \mathrm{e}-3$

$2.59 \mathrm{e}-3$

$0.00 \mathrm{e} 0$
Fig. 8 shows the equivalent stress distribution on the impeller under the part-load condition, it indicates that he equivalent stress on the impeller is uneven. Due to the ct of the flow field pressure, the equivalent stress gradcreases from the leading edge to the trailing edge, edge and hub, which is consistent with the location of gue failure in engineering. Meanwhile, the equivalent upon the pressure surface is larger than the suction fent thickness distribution, it concludes that the equivalent of the local thickening blade is much lower than unithickness blade. Hence, applying local thickening on ress. Importantly, the equivalent stress of the blade with ment thickness is lower than the decrement one. Therefore, it deduced that the fluid field can be improved by employing the increment thickness blade.

At the same time, the distribution of the total deformation as shown in Fig. 9, it illustrates that the deformation has the similar trend with the equivalent stress. The distribution of deformation on the impeller is asymmetrical, which is increasing with the rise of the radius, and reaches he maximum at the hub near the trailing edge. Considering the pressure of the flow field, the torsion and bend are generated upon the blade. Furthermore, the equivalent stress of the scheme 1 is larger than other schemes, which result in deformation distribution of the scheme 1 reaches the maximum.

Fig. 9, a) Total deformation distribution on the shrub under $0.2 Q$

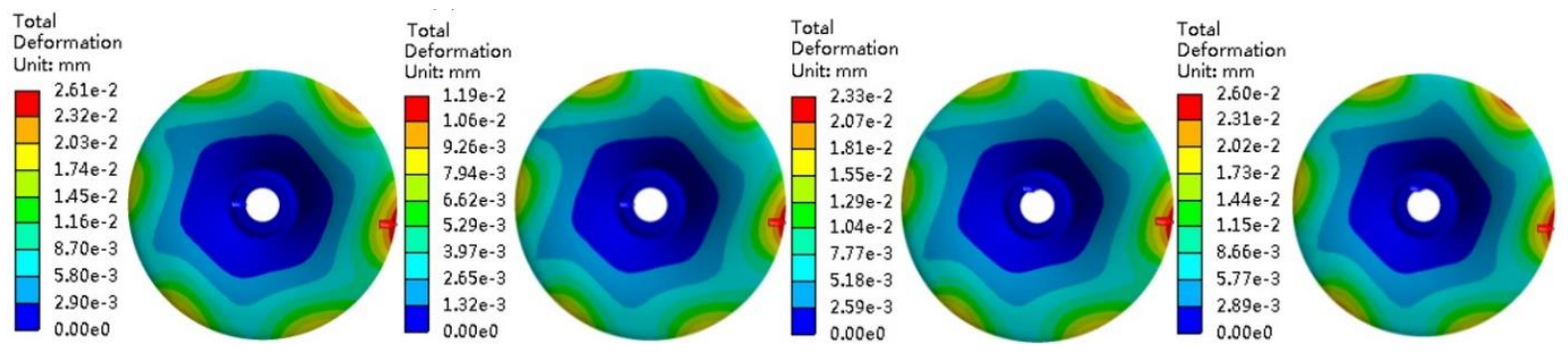

Fig. 9, b) Total deformation distribution on the hub under $0.2 Q$ 
In this paper, the material of the impeller employs the HT200, and the material properties is shown as follow: density $\rho=7800 \mathrm{~kg} / \mathrm{m}^{3}$, Poisson ratio $\mu=0.25$, elastic modulus $E=122 \mathrm{GPa}$ and the allowable stress $[\sigma]=80 \mathrm{MPa}$. In order to ensure the pump, operate under the safety and stability condition, the stress on the impeller should less than the allowable stress. It can be observed that the strength under the part-load condition satisfy the requirement of the structure.

\section{Experiment and verification}

In order to benchmark the accuracy of the simulation result, the hydraulic performance test of the scheme 3 was conducted test bench of I level accuracy at Jiangsu University, all experiment instruments are employed after calibration, the flow rate is measured by applying the turbine flowmeter with the precision of $\pm 1.0 \%$, at the same time, pressure sensor and torque-speed transducer with the precision of $\pm 0.5 \%$ are employed. According to the uncertainty analysis of the system, the comprehensive error of pump performance measurement is estimated to be within \pm $0.71 \%$, as shown in Fig. 10. All experiment equipment was calibrated before operation.

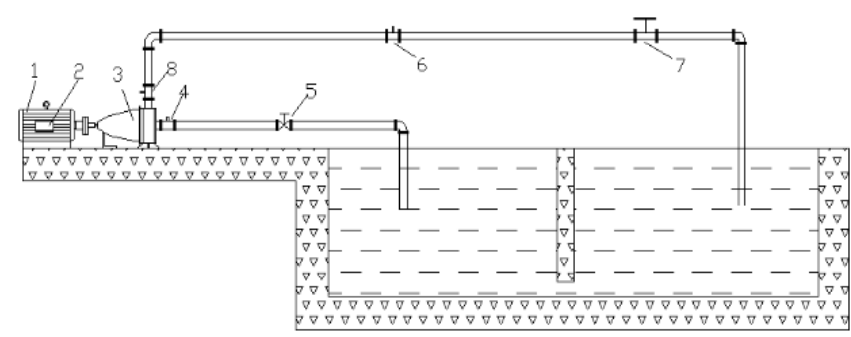

1 - Motor. 2 - Torque meter. 3 - Self-priming pump. 4 - Inlet pressure transducer. 5 - Inlet valve. 6 - Turbine flow rate meter. 7 - Outlet valve. 8 - Outlet pressure transducer

Fig. 10 Test rig

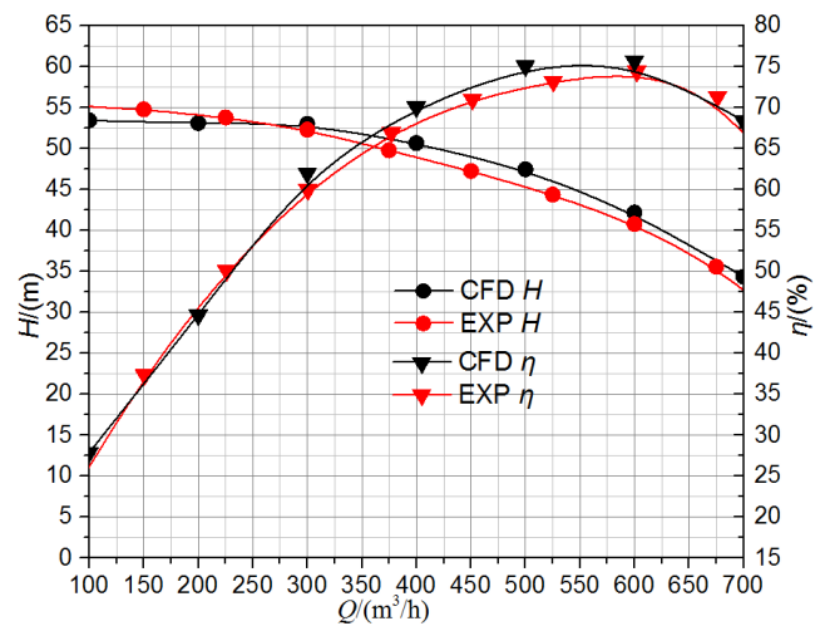

Fig. 11 Numerical and test results of external characteristic

As shown in Fig. 11, the hydraulic performance of simulation and test were compared, it can be seen that the energy performance curve obtained by numerical computation is more in line with test results under part load flow conditions $\left(Q=100 \mathrm{~m}^{3} / \mathrm{h}\right)$. Due to the mechanical loss of seals and bearings without considering in the numerical simulation, the deviations can be found between test and simu- lation, while the deviations are less than $3 \%$. Hence, the relatively accurate simulation result can be obtained by employing reasonable grid quality and turbulence model.

\section{Conclusions}

In this paper, in order to ensure the operation stability of the pump under the part-load condition, the energy performance, internal flow field and structural dynamic characteristics of the blades with different thickness distribution under $0.2 Q$ were theoretically and numerically investigated, and the conclusions can be obtained as follow:

1. The energy performance of the part-load condition cannot be improved by applying the uniform thickening on blade, but the local thickening blade can be achieved. And the internal flow field is improved by using increment thickness distribution blade, which is benefit to transform the kinetic energy into the potential energy.

2. The thickness distribution of blade exerts a significant role on the natural frequency of the impeller, due to the structural stiffness is improved by applying the thickening blade, the natural frequency of the thicker blade is obviously enhanced. Meanwhile, blade passing natural frequency is much lower than the natural frequency of the first mode, so it can effectively avoid the occurrence of resonance.

3. Compared with the equivalent stress of different thickness blade, it concluded that the equivalent stress of the local thickening blade is much lower than uniform thickness blade. Applying local thickening on the blade is an effective method to decrease the equivalent stress. And the total deformation of the impeller has the similar trend with the equivalent stress. Considering the material of the impeller employed the HT200, the stress on the blades are less than the allowable stress. Thus, the strength can satisfy the requirement of the structure.

4. The relevant test of the optimal blade was conducted to benchmark the results of the simulation, and the deviations between test and simulation are less than $3 \%$. Hence, the relatively accurate simulation result can be obtained by employing reasonable grid quality and turbulence model.

\section{Acknowledgements}

The authors gratefully acknowledge the support from the National Natural Science Foundation of China (No.51679111, No.51409127 and No.51579118), Six Talents Peak Project of Jiangsu Province JNHB-CXTD-005, Natural Science Foundation of Jiangsu Province (BE2016163, BRA2017353 and No. BK20161472), Priority Academic Program Development of Jiangsu Higher Education Institutions (PAPD) and National Key R\&D Program Project (No.2017YFC0403703)

\section{References}

1. Shigemitsu, T.; Fukutomi, J.; Nasada, R. 2011. The effect of blade outlet angle on performance and internal flow condition of mini turbo-pump, Journal of Thermal Science 20(1): 32-38. https://doi.org/10.1007/s11630-011-0431-3. 
2. Shigemitsu, T.; Fukutomi, J.; Nasada, R. 2011. Influence of blade outlet angle on impeller performance of mini turbo-pump, Turbomachinery 39: 112-118. https://doi.org/10.11458/tsj.39.112.

3. Li, W.; Zhao, X.; Li, W. 2017. Numerical prediction and Performance experiment in an engine cooling water pump with different blade outlet widths, Mathematical Problems in Engineering (6): 1-11. https://doi.org/10.1155/2017/8945712.

4. Wei, W.; Shi, W.; Jiang, X. 2016. Optimization design of multistage centrifugal pump impeller by orthogonal experiment and CFD, Journal of Drainage \& Irrigation Machinery Engineering 34(3): 191-197. https://doi.org/10.3969/j.issn.1674-8530.15.0035.

5. Chakraborty, S.; Biswas, N.; Choudhuri, K. 2013. CFD analysis of centrifugal pumps with variations of blade number at 3300 RPM, International Journal of Applied Engineering Research 8(3): 203-213.

6. Lei, T.; Zhu, B.; Cao, S. 2014. Influence of blade wrap angle on centrifugal pump performance by numerical and experimental study, Chinese Journal of Mechanical Engineering 27(1): 171-177. https://doi.org/10.3901/CJME.2014.01.171.

7. Derakhshan, S.; Mohammadi, B.; Nourbakhsh, A. 2010. The comparison of incomplete sensitivities and Genetic algorithms applications in 3D radial turbomachinery blade optimization, Computers \& Fluids 39(10): 2022-2029. https://doi.org/10.1016/j.compfluid.2010.07.003.

8. Wang, T.; Kong, F.; Xia, B. 2017. The method for determining blade inlet angle of special impeller using in turbine mode of centrifugal pump as turbine, Renewable Energy 109: 518-528. https://doi.org/10.1016/j.renene.2017.03.054.

9. Jeon, S. Y.; Kim, C. K.; Lee, S. M. 2017. Performance enhancement of a pump impeller using optimal design method, Journal of Thermal Science 26(2): 119-124. https://doi.org/10.1007/s11630-017-0919-6.

10. Tan, L.; Yu, Z.; Xu, Y. 2017. Role of blade rotational angle on energy performance and pressure fluctuation of a mixed-flow pump, Proceedings of the Institution of Mechanical Engineers Part a Journal of Power \& Energy 231(3): 227-238. https://doi.org/10.1177/0957650917689948.

11. Wang, C. 2012. Effect and experiment of different blade thickness on stainless steel stamping well pump performance, Transactions of the Chinese Society for Agricultural Machinery.

https://doi.org/10..6041/j.issn.1000-1298.2012.07.017.

H. Chang, W. Li, W. Shi, L. Wang, J. Liu

INFLUENCE OF BLADE THICKNESS DISTRIBUTION ON THE STRUCTURAL DYNAMIC CHARACTERISTICS UNDER PART-LOAD CONDITION

S u m m a r y

Due to the internal flow field is unstable under part-load condition, which has great influence on the structure strength of impeller. Therefore, in order to improve the operation stability, four different thickness distribution blades were proposed, the energy performance, internal flow field and structural dynamic characteristics under 0.2 $\mathrm{Q}$ were investigated based on the CFX and Workbench. The results show that the natural frequency is increased by thickening the blade, and the natural frequency of the blade with decrement thickness distribution is larger than the increment one. However, the equivalent stress of the local thickening blade is much lower than uniform thickness blade, that is, the internal flow field and strength of the impeller can be obviously improved by employing the increment thickness distribution. Finally, the test of the blade with increment thickness distribution was conducted to benchmark the accuracy of the simulation. This research could provide reference to the improvement of blade strength characteristics under the part-load condition.

Keywords: thickness distribution, structural dynamic characteristics, part-load condition.

Received September 03, 2018 Accepted February 15, 2019 